\title{
EXTREME INTEGRAL POLYNOMIALS ON A COMPLEX BANACH SPACE
}

\author{
SEÁN DINEEN*
}

\begin{abstract}
We obtain upper and lower set-theoretic inclusion estimates for the set of extreme points of the unit balls of $\mathscr{P}_{I}\left({ }^{n} E\right)$ and $\mathscr{P}_{N}\left({ }^{n} E\right)$, the spaces of $n$-homogeneous integral and nuclear polynomials, respectively, on a complex Banach space $E$. For certain collections of Banach spaces we fully characterise these extreme points. Our results show a difference between the real and complex space cases.
\end{abstract}

\section{Introduction}

Geometric properties of spaces of polynomials, e.g. smoothness, extreme points, exposed points, norm attaining polynomials, etc. have been investigated by a number of authors in recent years. We refer to [1], [3], [7], [8], [13], [12], [18], [20]. In particular, Ryan-Turett [18] and Boyd-Ryan [3] in their investigations examined the extreme points of the unit ball of the space of integral polynomials defined on a real Banach space. In this paper we study the extreme points of the unit ball of the space of integral polynomials defined on a complex Banach space. Throughout this paper $E$ will, unless otherwise stated, denote a Banach space over the complex numbers $C$. We let $B_{E}$ or $B$ denote the open unit ball of $E$. We refer to [11] for basic facts on polynomials on Banach spaces and to [9], [10] for the geometry of Banach spaces.

\section{Extreme Points}

If $E$ is a Banach space over $C$ we let

$\operatorname{Ext}_{R}(E)=\{x \in E:\|x\|=1,\|x+\lambda y\| \leq 1$ for $-1 \leq \lambda \leq 1$ implies $y=0\}$ and

$\operatorname{Ext}_{C}(E)=\{x \in E:\|x\|=1,\|x+\lambda y\| \leq 1$ for $|\lambda| \leq 1$ implies $y=0\}$.

* The author wishes to thank Bogdan Grecu and Manolo Maestre for helpful remarks.

Received September 20, 2000. 
Thus, $\operatorname{Ext}_{R}(E)$ is the set of all (real) extreme points of the unit ball of $E$ and $\operatorname{Ext}_{C}(E)$ is the set of complex extreme points of the same set. Clearly $\operatorname{Ext}_{R}(E) \subset \operatorname{Ext}_{C}(E)$. The inclusion may be proper since $\operatorname{Ext}_{R}\left(L^{1}(0,1)\right)$ is empty while $\operatorname{Ext}_{C}\left(L^{1}(0,1)\right)$ consists of all unit vectors. if

A Banach space is strictly convex (or rotund) (respectively strictly $c$-convex)

$$
\operatorname{Ext}_{R}(E)=\{x \in E:\|x\|=1\}
$$

(respectively $\operatorname{Ext}_{C}(E)=\{x \in E:\|x\|=1\}$ ). As noted above the Banach space $L^{1}(0,1)$ is strictly $c$-convex but is not strictly convex.

The role of extreme points in functional analysis, convexity theory, linear programming and optimisation theory is well documented. Complex extreme points were introduced by Thorpe and Whitley [21] in order to prove a strong maximum modulus principle for Banach-valued holomorphic functions. Since their introduction they have proved useful in the study of the Shilov boundary, complex geodesics, invariant metrics, bounded symmetric domains and $\mathrm{JB}^{*}$ triple systems. We refer to [2], [4], [5], [14], [15], [22], [23] for details.

\section{Integral and Nuclear Polynomials}

If $E$ is a complex Banach space $\mathscr{P}\left({ }^{n} E\right)$ denotes the space of continuous $n$ homogeneous polynomials on $E$ endowed with the norm $\|\cdot\|:=\|\cdot\|_{B}$ of uniform convergence over the unit ball $B$ of $E$. A polynomial $P \in \mathscr{P}\left({ }^{n} E\right)$ is said to be integral if there exists a regular Borel measure $\mu$ on the unit ball of $E^{\prime}, B_{E^{\prime}}$ endowed with the weak* topology, such that

$$
P(x)=\int_{B_{E^{\prime}}} \phi(x)^{n} d \mu(\phi)
$$

for all $x$ in $E$. We let $\mathscr{P}_{I}\left({ }^{n} E\right)$ denote the space of all $n$-homogeneous integral polynomials on $E$ and we endow this space with the norm $\|\cdot\|_{I}:=\inf \|\mu\|$ where the infimum is taken over all $\mu$ satisfying (1).

An $n$-homogeneous polynomial $P$ on $E$ is nuclear if there exists a bounded sequence $\left(\phi_{j}\right)_{j} \subset E^{\prime}$ and $\left(\lambda_{j}\right)_{j} \in l_{1}$ such that

$$
P(x)=\sum_{j=1}^{\infty} \lambda_{j} \phi_{j}(x)^{n}
$$

for all $x$ in $E$. The space of all nuclear $n$-homogeneous polynomials on $E$, $\mathscr{P}_{N}\left({ }^{n} E\right)$, is a Banach space when $\|P\|_{N}$ is defined to be the infimum of $\sum_{j=1}^{\infty}\left|\lambda_{j}\right|\left\|\phi_{j}\right\|^{n}$ taken over all representations of $P$ satisfying (2).

Proposition 3.1. Let E denote a complex Banach space. 
(a) The space $\mathscr{P}_{I}\left({ }^{n} E\right)$ is isometrically isomorphic to $(\widehat{\otimes} E)^{\prime}$.

(b) $\mathscr{P}_{N}\left({ }^{n} E\right) \subset \mathscr{P}_{I}\left({ }^{n} E\right) \subset \mathscr{P}\left({ }^{n} E\right)$ and $\|\cdot\| \leq\|\cdot\|_{I} \leq\|\cdot\|_{N}$.

(c) If $\phi \in E^{\prime}$ then $\phi^{n} \in \mathscr{P}_{N}\left({ }^{n} E\right)$ and

$$
\|\phi\|^{n}=\left\|\phi^{n}\right\|_{I}=\left\|\phi^{n}\right\|_{N}
$$

(d) If $E^{\prime}$ has the approximation property then $\mathscr{P}_{N}\left({ }^{n} E\right)$ is isometrically isomorphic to $\widehat{\otimes} E^{\prime}$ and $\mathscr{P}_{N}\left({ }^{n} E\right)^{\prime}=\mathscr{P}\left({ }^{n} E^{\prime}\right)$ isometrically.

(e) If $\ell_{1} \hookrightarrow \widehat{\bigotimes}_{n, s, \epsilon} E$ (in particular if $E^{\prime}$ has the Radon-Nikodým Property) then $\mathscr{P}_{I}\left({ }^{n} E\right)$ and $\mathscr{P}_{N}\left({ }^{n} E\right)$ are isometrically isomorphic.

(f) $\operatorname{Ext}_{R}\left(\mathscr{P}_{I}\left({ }^{n} E\right)\right) \subset\left\{\phi^{n}: \phi \in E^{\prime},\|\phi\|=1\right\}$.

(g) $\operatorname{Ext}_{R}\left(\mathscr{P}_{I}\left({ }^{n} E\right)\right) \subset \operatorname{Ext}_{R}\left(\mathscr{P}_{N}\left({ }^{n} E\right)\right)$.

Proof. Parts (a), (b), (c) and (d) are well known. Parts (e) and (f) are due independently to C. Boyd-R. A. Ryan [3] and D. Carando-V. Dimant [6] while (g) can be deduced from the proof of [3, Theorem 2].

Remarks. (i) By (b) and (g) we have

$$
\mathscr{P}_{N}\left({ }^{n} E\right) \subset \mathscr{P}_{I}\left({ }^{n} E\right) \quad \text { and } \quad \operatorname{Ext}_{R}\left(\mathscr{P}_{I}\left({ }^{n} E\right)\right) \subset \operatorname{Ext}_{R}\left(\mathscr{P}_{N}\left({ }^{n} E\right)\right) \text {. }
$$

This contrasting information is quite useful since $\operatorname{Ext}_{R}\left(\mathscr{P}_{I}\left({ }^{n} E\right)\right)$ has good abstract properties while calculations are easier with elements of $\operatorname{Ext}_{R}\left(\mathscr{P}_{N}\left({ }^{n} E\right)\right)$.

(ii) In general we do not know if $\mathscr{P}_{N}\left({ }^{n} E\right)$ is a dual Banach space but, by (a) and (g), $\operatorname{Ext}_{R}\left(\mathscr{P}_{N}\left({ }^{n} E\right)\right)$ is non-empty.

For real Banach space we also have the following result which is in contrast to the results we obtain in the next section for complex Banach spaces.

Proposition 3.2 ([3], [18]). If $E$ is a reflexive Banach space over $\mathrm{R}$ and $n>1$ then

$$
\operatorname{Ext}_{R}\left(\mathscr{P}_{I}\left({ }^{n} E\right)\right)=\left\{ \pm \phi^{n}: \phi \in E^{\prime},\|\phi\|=1\right\} .
$$

\section{Extreme Polynomials}

Proposition 4.1. If $E$ is a complex Banach space and $n \geq 1$ then

$$
\left\{\phi \in E^{\prime}: \phi^{n} \in \operatorname{Ext}_{R}\left(\mathscr{P}_{N}\left({ }^{n} E\right)\right)\right\} \subset \operatorname{Ext}_{C}\left(E^{\prime}\right) .
$$


Proof. Suppose $\|\phi\|=1$ and $\phi \notin \operatorname{Ext}_{C}\left(E^{\prime}\right)$. Then there exists $\psi \in E^{\prime}$, $\psi \neq 0$, such that $\|\phi+\lambda \psi\| \leq 1$ for all $\lambda \in \mathrm{C},|\lambda| \leq 1$. If $\omega=e^{\frac{2 \pi i}{n+1}}$ then

$$
\sum_{j=0}^{n}\left(\phi+\omega^{j} \psi\right)^{n}=\sum_{k=0}^{n}\left(\begin{array}{l}
n \\
k
\end{array}\right) \psi^{k} \phi^{n-k}\left(\sum_{j=0}^{n} \omega^{k j}\right) .
$$

If $k \neq 0$ then $\sum_{j=0}^{n} \omega^{k j}=0$. Hence

$$
\frac{1}{n+1} \sum_{j=0}^{n}\left(\phi+\omega^{j} \psi\right)^{n}=\phi^{n} .
$$

Since $\|\phi\|=1$ and $\left\|\phi+\omega^{j} \psi\right\| \leq 1$ for all $j$, Proposition 3.1(c) implies $\left\|\phi^{n}\right\|_{N}=1$ and, $\left\|\left(\phi+\omega^{j} \psi\right)^{n}\right\|_{N} \leq 1$ for all $j$. Hence $\phi^{n} \notin \operatorname{Ext}_{R}\left(\mathscr{P}_{N}\left({ }^{n} E\right)\right)$. This completes the proof.

If $E$ is a complex Banach space we say that $x$ and $y$ in $E$ are equivalent if there exists $\alpha \in \mathrm{C},|\alpha|=1$, such that $x=\alpha y$. If $A \subset E$ we denote by $\diamond[A]$ the cardinality of the set of equivalence classes in $A$. For example it is well known that $\diamond\left[\operatorname{Ext}_{R}\left(\ell_{1}^{n}\right)\right]=n$. For complex extreme points we obtain a different type of result.

Corollary 4.2. If $E$ is a finite dimensional complex Banach space of dimension $\geq 2$ then

$$
\diamond\left[\operatorname{Ext}_{C}(E)\right]=\infty
$$

Proof. By Proposition 4.1 and the Krein-Milman Theorem

$$
\operatorname{dim}\left(\mathscr{P}_{I}\left({ }^{n} E^{\prime}\right)\right) \leq \diamond\left[\operatorname{Ext}_{C}(E)\right]
$$

for any positive integer $n$. Since the monomials $z^{j} w^{n-j}, j=1,2, \ldots, n$ are linearly independent, $\operatorname{dim}\left(\mathscr{P}_{I}\left({ }^{n} E^{\prime}\right)\right)=\operatorname{dim}\left(\mathscr{P}\left({ }^{n} E^{\prime}\right)\right)$ is at least $n$. Hence $\diamond\left[\operatorname{Ext}_{C}(E)\right] \geq n$ for all $n$. This completes the proof.

If $E$ is a Banach space we say that $A \subset E^{\prime}$ is $E$-transitive if for all $\theta, \phi$ in $A$ there exists an isometry of $E$ onto itself, $T$, such that $\phi \circ T=\theta$, that is ${ }^{t} T(\phi)=\theta$ where ${ }^{t} T$ denotes the transpose of $T$.

COROLlary 4.3. If the set of complex extreme points of the unit ball of $E^{\prime}$ is E-transitive then

$$
\operatorname{Ext}_{R}\left(\mathscr{P}_{I}\left({ }^{n} E\right)\right)=\left\{\phi^{n}: \phi \in E^{\prime}, \phi \in \operatorname{Ext}_{C}\left(E^{\prime}\right)\right\}
$$

and, in particular,

$$
\operatorname{Ext}_{R}\left(E^{\prime}\right)=\operatorname{Ext}_{C}\left(E^{\prime}\right)
$$


Proof. By Proposition 3.1(a), (f) and (g) and Proposition 4.1, $\operatorname{Ext}_{R}\left(\mathscr{P}_{I}\left({ }^{n} E\right)\right)$ contains an element $\phi^{n}$ where $\phi \in \operatorname{Ext}_{C}\left(E^{\prime}\right)$. Let $\psi \in \operatorname{Ext}_{C}\left(E^{\prime}\right)$. By $E$ transitivity of the set of complex extreme points there exists an isometry $T$ of $E$ such that $\psi \circ T=\phi$. If $P \in \mathscr{P}_{I}\left({ }^{n} E\right)$ and $\left\|\psi^{n} \pm P\right\|_{I} \leq 1$ then $\left\|\psi^{n} \circ T \pm P \circ T\right\|_{I} \leq 1$. Since $\psi^{n} \circ T=(\psi \circ T)^{n}=\phi^{n}$ and $\phi^{n} \in \operatorname{Ext}_{R}\left(\mathscr{P}_{I}\left({ }^{n} E\right)\right)$ this implies $P \circ T=0$. Hence $P=0$ and $\psi^{n} \in \operatorname{Ext}_{R}\left(\mathscr{P}_{I}\left({ }^{n} E\right)\right)$. An application of Proposition 4.1 completes the proof.

Example 4.4. Let $\mathscr{A}$ denote a $\mathscr{W}^{*}$-algebra, i.e. a $\mathscr{C}^{*}$-algebra which is also a dual Banach space. We can suppose, without loss of generality, that $\mathscr{A}$ is a $\mathscr{C}^{*}$-subalgebra of $\mathscr{B}(\mathscr{H})$, the space of bounded linear operators on the Hilbert space $\mathscr{H}$. By [15] the real and complex extreme points of the unit ball of a $\mathscr{C}^{*}$-algebra coincide.

Let

$$
\mathscr{A}^{\perp}=\{w \in \mathscr{N}(\mathscr{H}): \operatorname{trace}(v w)=0 \text { for all } v \in \mathscr{A}\} .
$$

where $\mathscr{N}(\mathscr{H})$ is the space of trace class operators on $\mathscr{H}$. By [17, Theorem 4.2.9], $\mathscr{A}_{*}:=\mathscr{N}(\mathscr{H}) / \mathscr{A}^{\perp}$ is the unique isometric predual of $\mathscr{A}$. The $\left(\mathscr{A}, \mathscr{A}_{*}\right)$ duality is given by

$$
\left(u, w+\mathscr{A}^{\perp}\right)=\operatorname{trace}(u w)=\sum_{x \in E}\langle u w(x), x\rangle
$$

where $u \in \mathscr{A}, w+\mathscr{A}^{\perp} \in \mathscr{A}_{*}$ and $E$ is an orthonormal basis for $\mathscr{H}$. Let $\mathscr{U}$ denote the set of unitary elements in $\mathscr{A}$, i.e.

$$
\mathscr{U}=\left\{u \in \mathscr{A}: u u^{*}=u^{*} u=1_{\mathscr{H}}\right\} .
$$

Clearly $u w \in \mathscr{A}^{\perp}$ for $u$ unitary in $\mathscr{A}$ and $w$ in $\mathscr{A}^{\perp}$. Hence the mapping

$$
u_{t}: \mathscr{A}_{*} \hookrightarrow \mathscr{A}_{*}, u_{t}\left(v+\mathscr{A}^{\perp}\right):=u v+\mathscr{A}^{\perp}
$$

is well defined and easily seen to be an isometry of $\mathscr{A}_{*}$. If $v_{1}$ and $v_{2}$ are unitary elements in $\mathscr{A}$ then $v_{2}^{*} v_{1}$ is also unitary and for all $w+\mathscr{A}^{\perp} \in \mathscr{A}_{*}$ we have

$$
\begin{aligned}
\left(v_{2} \circ\left(v_{2}^{*} v_{1}\right)_{t}, w+\mathscr{A}^{\perp}\right) & =\left(v_{2}, v_{2}^{*} v_{1} w+\mathscr{A}^{\perp}\right)=\sum_{x \in E}\left\langle v_{2} v_{2}^{*} v_{1} w(x), x\right\rangle \\
& =\sum_{x \in E}\left\langle v_{1} w(x), x\right\rangle=\left(v_{1}, w+\mathscr{A}^{\perp}\right) .
\end{aligned}
$$

Hence

$$
v_{1}=v_{2} \circ\left(v_{2}^{*} v_{1}\right)_{t}
$$


and $\mathscr{U}$ is $\mathscr{A}_{*}$-transitive. Results in [16] (see also Example 4.6) imply that

$$
\operatorname{Ext}_{C}(\mathscr{A})=\operatorname{Ext}_{R}(\mathscr{A})=\mathscr{U}
$$

i.e. the real (and complex) extreme points coincide with the set of unitary elements when $\mathscr{A}$ is one of the following :

(a) a commutative $\mathscr{W}^{*}$-algebra,

(b) $\mathscr{B}(\mathscr{H}), \mathscr{H}$ finite dimensional,

(c) a type $\mathrm{II}_{1}$ factor.

Hence, by Corollary 4.3 , if $\mathscr{A}$ is any of the above $\mathscr{W}^{*}$-algebras then

$$
\operatorname{Ext}_{R}\left(\mathscr{P}_{I}\left(\mathscr{A}_{*}\right)\right)=\left\{\phi^{n}: \phi \in \operatorname{Ext}_{C}(\mathscr{A})\right\} .
$$

If $\operatorname{dim}(\mathscr{A}) \geq 2$ then $\operatorname{Ext}_{C}(\mathscr{A})$ is a proper subset of $\{x: x \in \mathscr{A},\|x\|=1\}$. (See for instance [5, Theorem 1.9]). For example if $\mathscr{A}=\ell_{\infty}^{m}$ then its predual is $\ell_{1}^{m}$ and $\operatorname{Ext}_{R}\left(\mathscr{P}_{I}\left({ }^{n} \ell_{1}^{m}\right)\right)$ consists of all $\phi^{n}$ where $\phi$ lies in the distinguished boundary of the unit polydisc in $\mathrm{C}^{m}$.

The above method does not extend to all $\mathscr{W}^{*}$-algebras. By [16, Corollary 2]

$$
\operatorname{Ext}_{C}(\mathscr{B}(\mathscr{H}))=\left\{u \in \mathscr{B}(\mathscr{H}): u u^{*}=I_{\mathscr{H}} \text { or } u^{*} u=I_{\mathscr{H}}\right\} .
$$

If $\mathscr{H}$ is infinite dimensional then the forward shift, $S$, is a non-unitary extreme point of the unit ball of $\mathscr{B}(\mathscr{H})$. By [16] isometries of $\mathscr{B}(\mathscr{H})$ map extreme points to extreme points and unitaries to unitaries. Hence no isometry of $\mathscr{B}(\mathscr{H})$ maps $S$ to $I_{\mathscr{H}}$ and $\operatorname{Ext}_{C}(\mathscr{B}(\mathscr{H}))=\operatorname{Ext}_{R}(\mathscr{B}(\mathscr{H}))$ is not $\mathscr{B}(\mathscr{H})_{*}$-transitive.

Proposition 4.5. If $E$ is a finite dimensional complex Banach space then

$$
\left\{\phi^{n}: \phi \in \operatorname{Ext}_{R}\left(E^{\prime}\right)\right\} \subset \operatorname{Ext}_{R}\left(\mathscr{P}_{I}\left({ }^{n} E\right)\right)
$$

for all $n$.

Proof. Let $\phi \in \operatorname{Ext}_{R}\left(E^{\prime}\right)$. Suppose $\phi^{n} \notin \operatorname{Ext}_{R}\left(\mathscr{P}_{I}\left({ }^{n} E\right)\right)$. Since $E$ is finite dimensional Proposition 3.1(e) implies that $\phi^{n} \notin \operatorname{Ext}_{R}\left(\mathscr{P}_{N}\left({ }^{n} E\right)\right)$.

Hence there exist $P$ and $Q$ in $\mathscr{P}_{N}\left({ }^{n} E\right), P \neq Q$, and $\lambda \in \mathrm{R}, 0<\lambda<1$, such that

$$
\phi^{n}=\lambda P+(1-\lambda) Q
$$

and

$$
\|P\|=\|Q\|=1 .
$$

Since $E$ is finite dimensional so also is $\mathscr{P}_{N}\left({ }^{n} E\right)$. Hence the unit ball of $\mathscr{P}_{N}\left({ }^{n} E\right)$ is the convex hull of its extreme points and thus, by Proposition 3.1(f), there exists $\left(\phi_{i}\right)_{i=1}^{k+l} \subset E^{\prime}, \phi_{i} \neq 0$, such that $\lambda P=\sum_{i=1}^{k} \phi_{i}^{n},\|\lambda P\|_{N}=$ $\sum_{i=1}^{k}\left\|\phi_{i}^{n}\right\|,(1-\lambda) Q=\sum_{i=k+1}^{k+l} \phi_{i}^{n}$ and $\|(1-\lambda) Q\|_{N}=\sum_{i=k+1}^{k+l}\left\|\phi_{i}^{n}\right\|$. 
Hence

$$
\left\|\phi^{n}\right\|_{N}=\left\|\phi^{n}\right\|=1=\sum_{i=1}^{k+l}\left\|\phi_{i}^{n}\right\|
$$

Choose $x_{0} \in E,\left\|x_{0}\right\|=1$, such that $\phi\left(x_{0}\right)=1$. On differentiating $\phi^{n}=$ $\sum_{i=1}^{k+l} \phi_{i}^{n}$ at $x_{0}$ we obtain

$$
\phi=\phi^{n-1}\left(x_{0}\right) \phi=\sum_{i=1}^{k+l} \phi_{i}^{n-1}\left(x_{0}\right) \phi_{i} .
$$

Hence

$$
1=\|\phi\| \leq \sum_{i=1}^{k+l}\left|\phi_{i}^{n-1}\left(x_{0}\right)\right|\left\|\phi_{i}\right\| \leq \sum_{i=1}^{k+l}\left\|\phi_{i}\right\|^{n}=1 .
$$

and we can choose for each $i, \beta_{i} \in \mathrm{C}$, with $\left|\beta_{i}\right|=1$ such that

$$
\phi=\sum_{i=1}^{k+l}\left|\phi_{i}^{n-1}\left(x_{0}\right)\right| \cdot\left\|\phi_{i}\right\| \cdot \frac{\beta_{i} \phi_{i}}{\left\|\phi_{i}\right\|} .
$$

Since $\phi \in \operatorname{Ext}_{R}\left(E^{\prime}\right)$ this implies that for each $i, \phi_{i}=\alpha_{i} \phi$ for some $\alpha_{i} \in \mathrm{C}$. Hence $P=\phi^{n}=Q$. This contradicts our hypothesis and shows that $\phi^{n} \in$ $\operatorname{Ext}_{R}\left(\mathscr{P}_{N}\left({ }^{n} E\right)\right)=\operatorname{Ext}_{R}\left(\mathscr{P}_{I}\left({ }^{n} E\right)\right)$ and completes the proof.

ExAmple 4.6. If $\mathscr{J}$ is a $\mathrm{JB}^{*}$-algebra then, by [4, Lemma 4.1] and [15, Theorem 11],

$$
\operatorname{Ext}_{R}(\mathscr{J})=\operatorname{Ext}_{C}(\mathscr{J})
$$

Hence Propositions 4.1 and 4.5 imply

$$
\left\{\phi^{n}: \phi \in \operatorname{Ext}_{R}(\mathscr{J})\right\}=\operatorname{Ext}_{R}\left(\mathscr{P}_{I}\left({ }^{n} \mathscr{J}^{\prime}\right)\right)
$$

for any finite dimensional $\mathrm{JB}^{*}$-algebra $\mathscr{J}$. This result gives an independent proof of the result in Example 4.4 for finite dimensional $\mathscr{C}^{*}$-algebras.

EXAMPLE 4.7. If $E^{\prime}$ is a strictly convex finite dimensional Banach space then

$$
\operatorname{Ext}_{R}\left(\mathscr{P}_{I}\left({ }^{n} E\right)\right)=\left\{\phi^{n}: \phi \in E^{\prime},\|\phi\|=1\right\}
$$

This is similar to the result for real Banach spaces quoted above (Proposition 3.2).

To extend Proposition 4.5 to infinite dimensional spaces we require weak ${ }^{*}$ exposed points. For convenience we introduce these in complex form.

Definition 4.8. Let $E$ denote a Banach over C. A linear functional $\phi \in E^{\prime}$ is a weak ${ }^{*}$-exposed point of the unit ball of $E^{\prime}$ if $\|\phi\|=1$ and there exists 
$x \in E,\|x\|=1$, such that $\Re(\phi(x))=1$ and $\Re(\psi(x))<1$ for all $\psi \in E^{\prime}$, $\|\psi\| \leq 1$ and $\psi \neq \phi$. We say that $x$ weak $^{*}$-exposes $\phi$. We let $\operatorname{Exp}_{\omega^{*}}\left(E^{\prime}\right)$ denote the set of all weak*-exposed points of the unit ball of $E^{\prime}$.

If $\phi: E \longrightarrow C$ is complex linear then $\tilde{\phi}:=\Re(\phi)$ is a real linear mapping. Moreover, $\|\phi\|=\|\tilde{\phi}\|$ and $\phi(x)=\tilde{\phi}(x)-i \tilde{\phi}(i x)$ for all $x$ in $E$. Hence if $\phi_{1}$ and $\phi_{2}$ are complex linear then $\phi_{1}=\phi_{2}$ if and only if $\Re\left(\phi_{1}\right)=\Re\left(\phi_{2}\right)$.

If $x$ weak ${ }^{*}$-exposes $\phi$ and $\phi=\frac{\phi_{1}+\phi_{2}}{2}$ with $\left\|\phi_{1}\right\|=\left\|\phi_{2}\right\|=1$ then, $\left|\phi_{1}(x)\right| \leq$ 1 and $\left|\phi_{2}(x)\right| \leq 1$ imply $\phi_{1}(x)=\phi_{2}(x)=1$. Hence $\phi_{1}=\phi_{2}=\phi$ and $\operatorname{Exp}_{\omega^{*}}\left(E^{\prime}\right) \subset \operatorname{Ext}_{R}\left(E^{\prime}\right)$.

For our next result, which was motivated by [3, Proposition 5], we require the following Lemma [3, Lemma 4].

LemMa 4.9. Let $E$ be a normed space and let $\phi$ be a unit vector in $E^{\prime}$. Suppose that for each finite dimensional subspace $F$ of $E$ there exists a subspace $G$ of $E, F \subset G$, such that $\left.\phi\right|_{G}$ is an extreme point of the unit ball of $G^{\prime}$. Then $\phi$ is an extreme point of the unit ball of $E^{\prime}$.

Proposition 4.10. If $E$ is a complex Banach space then

$$
\left\{\phi^{n}: \phi \in \operatorname{Exp}_{\omega^{*}}\left(E^{\prime}\right)\right\} \subset \operatorname{Ext}_{R}\left(\mathscr{P}_{I}\left({ }^{n} E\right)\right) .
$$

Proof. Let $\phi \in \operatorname{Exp}_{\omega^{*}}\left(E^{\prime}\right)$ and suppose $x$ weak ${ }^{*}$-exposes $\phi$. Given a finite dimensional subspace $X$ of $\bigotimes_{n, s, \epsilon} E$ choose a finite dimensional subspace $F$ of $E$ such that $x \in F$ and $X \subset \bigotimes_{n, s, \epsilon} F$. Since $x \in F,\left\|\left.\phi\right|_{F}\right\|=1$. Let $\psi \in F^{\prime},\|\psi\|=1$ and $\psi \neq\left.\phi\right|_{F}$. By the Hahn-Banach Theorem there exists $\tilde{\psi} \in E^{\prime}$ such that $\left.\tilde{\psi}\right|_{F}=\psi$ and $\|\tilde{\psi}\|=\|\psi\|=1$. Since $\tilde{\psi} \neq \phi$ we have $\Re(\tilde{\psi}(x))=\Re(\psi(x))<1$ and $\left.\phi\right|_{F} \in \operatorname{Exp}_{\omega^{*}}\left(F^{\prime}\right) \subset \operatorname{Ext}_{R}\left(F^{\prime}\right)$.

By Proposition 4.5

$$
\left(\left.\phi\right|_{F}\right)^{n}=\left.\phi^{n}\right|_{F} \in \operatorname{Ext}_{R}\left(\mathscr{P}_{I}\left({ }^{n} F\right)\right)=\operatorname{Ext}_{R}\left(\left(\bigotimes_{n, s, \epsilon} F\right)^{\prime}\right) .
$$

By Proposition 3.1(a) and Lemma 4.9,

$$
\phi^{n} \in \operatorname{Ext}_{R}\left(\left(\bigotimes_{n, s, \epsilon} E\right)^{\prime}\right)=\operatorname{Ext}_{R}\left(\mathscr{P}_{I}\left({ }^{n} E\right)\right) .
$$

This completes the proof.

EXAmPLe 4.11. If $E$ is a complex Banach space let $D(E)=\{x \in E:\|x\|=1$ and $f(\cdot):=\|\cdot\|$ is real Gâteaux differentiable at $x\}$. 
Then, see [19] and [10],

$$
\operatorname{Exp}_{\omega^{*}}\left(E^{\prime}\right)=\left\{\phi \in E^{\prime}: \phi=f^{\prime}(x) \text { for some } x \in D(E)\right\} \text {. }
$$

For many classical Banach spaces, e.g. $\ell_{p}, 1<p<\infty$, this result can be used to show that

$$
\left\{\phi: \phi \in E^{\prime},\|\phi\|=1\right\}=\operatorname{Exp}_{\omega^{*}}\left(E^{\prime}\right)
$$

and hence, by Proposition 4.10, that

$$
\operatorname{Ext}_{R}\left(\mathscr{P}_{I}\left({ }^{n} E\right)\right)=\left\{\phi^{n}: \phi \in E^{\prime},\|\phi\|=1\right\} .
$$

If $E=\ell_{1}$ then $x=\left(x_{n}\right)_{n} \in D\left(\ell_{1}\right)$ if and only if $x_{n} \neq 0$ for all $n$. Hence $\phi:=\left(y_{n}\right)_{n} \in \operatorname{Exp}_{\omega^{*}}\left(\ell_{\infty}\right)$ if and only if $\left|y_{n}\right|=1$ for all $n$ and $\operatorname{Exp}_{\omega^{*}}\left(\ell_{\infty}\right)=$ $\operatorname{Ext}_{R}\left(\ell_{\infty}\right)$. Since $\operatorname{Ext}_{R}\left(\ell_{\infty}\right)=\operatorname{Ext}_{C}\left(\ell_{\infty}\right)$ this implies

$$
\left\{\phi^{n}: \phi \in \operatorname{Ext}_{R}\left(\ell_{\infty}\right)\right\}=\operatorname{Ext}_{R}\left(\mathscr{P}_{I}\left({ }^{n} \ell_{1}\right)\right)
$$

and we recover a special case of Example 4.4 since $\ell_{\infty}$ is a $\mathscr{W}^{*}$-algebra.

ExAmPLE 4.12. Let $\ell_{\infty}^{m}$ denote $\mathrm{C}^{m}$ endowed with the supremum norm. By Proposition 3.1(f)

$$
\operatorname{Ext}_{R}\left(\mathscr{P}_{I}\left({ }^{n} \ell_{\infty}^{m}\right)\right) \subset\left\{\phi^{n}: \phi \in \ell_{1}^{m},\|\phi\|=1\right\}
$$

for $n \geq 2$. If $\phi:=\left(w_{1}, \ldots, w_{m}\right) \in \ell_{1}^{m}, \theta:=\left(\theta_{1}, \ldots, \theta_{m}\right) \in \mathbf{R}^{m}$ and $\sigma$ is a permutation of $\{1, \ldots, m\}$ let

$$
\phi_{\theta, \sigma}:=\left(e^{i \theta_{1}} w_{\sigma(1)}, \ldots, e^{i \theta_{m}} w_{\sigma(m)}\right) .
$$

It is easily seen that $\phi_{\theta, \sigma}^{n} \in \operatorname{Ext}_{R}\left(\mathscr{P}_{I}\left({ }^{n} \ell_{\infty}^{m}\right)\right)$ if and only if $\phi^{n} \in \operatorname{Ext}_{R}\left(\mathscr{P}_{I}\left({ }^{n} \ell_{\infty}^{m}\right)\right)$. Hence to show

$$
\operatorname{Ext}_{R}\left(\mathscr{P}_{I}\left({ }^{n} \ell_{\infty}^{m}\right)\right)=\left\{\phi^{n}: \phi \in \ell_{1}^{m},\|\phi\|=1\right\}
$$

it suffices to show that $\alpha^{n} \in \operatorname{Ext}_{R}\left(\mathscr{P}_{I}\left({ }^{n} \ell_{\infty}^{m}\right)\right)$ where $\alpha:=\left(\alpha_{1}, \ldots, \alpha_{m}\right)$ satisfies $0 \leq \alpha_{1} \leq \alpha_{2} \leq \cdots \leq \alpha_{m}$ and $\sum_{i=1}^{m} \alpha_{i}=1$. Let $l$ denote the smallest positive integer such that $\alpha_{l}>0$. If $l=m$ then $\alpha \in \operatorname{Ext}_{R}\left(\ell_{1}^{m}\right)$ and Proposition 4.5 implies that $\alpha^{n} \in \operatorname{Ext}_{R}\left(\mathscr{P}_{I}\left({ }^{n} \ell_{\infty}^{m}\right)\right)$. We may thus suppose, from now on, that $l<m$.

By Proposition 3.1(d), $\left(\mathscr{P}_{I}\left({ }^{n} \ell_{\infty}^{m}\right)\right)^{\prime}=\mathscr{P}\left({ }^{n} \ell_{1}^{m}\right)$ with duality $\left\langle\phi^{n}, P\right\rangle=$ $P(\phi)$ for $P \in \mathscr{P}\left({ }^{n} \ell_{1}^{m}\right)$ and $\phi \in\left(\ell_{\infty}^{m}\right)^{\prime}=\ell_{1}^{m}$. By the Krein-Milman Theorem $|P|, P \in \mathscr{P}\left({ }^{n} \ell_{1}^{m}\right)$, achieves its maximum over the unit ball of $\ell_{1}^{m}$ at a point $\phi$ where $\phi^{n} \in \operatorname{Ext}_{R}\left(\mathscr{P}_{I}\left({ }^{n} \ell_{\infty}^{m}\right)\right)$. 
Now consider the 2-homogeneous polynomial on $\ell_{1}^{m}$

$$
P_{\alpha}\left(z_{1}, \ldots, z_{m}\right):=\sum_{l \leq i<j \leq m} z_{i} z_{j}+\sum_{k \geq l} \frac{\alpha_{k}-\alpha_{l}}{2 \alpha_{k}} z_{k}^{2} .
$$

Since

$$
\begin{aligned}
\left|P_{\alpha}\left(z_{1}, \ldots, z_{m}\right)\right| & =\left|P_{\alpha}\left(0, \ldots, 0, z_{l}, \ldots, z_{m}\right)\right| \\
& \leq \sum_{l \leq i<j \leq m}\left|z_{i}\right|\left|z_{j}\right|+\sum_{k \geq l} \frac{\alpha_{k}-\alpha_{l}}{2 \alpha_{k}}\left|z_{k}\right|^{2} \\
& =P_{\alpha}\left(0, \ldots, 0,\left|z_{l}\right|, \ldots,\left|z_{m}\right|\right) \\
& \leq\left\|P_{\alpha}\right\| \cdot\left(\sum_{k \geq l}\left|z_{k}\right|\right)^{2}
\end{aligned}
$$

it follows that $\left|P_{\alpha}\right|$ achieves its maximum, $\left\|P_{\alpha}\right\|:=\left\|P_{\alpha}\right\|_{B_{\ell_{1}^{m}}}$, at some point which has the form $e^{i \theta}\left(0, \ldots, 0, x_{l}, \ldots, x_{m}\right)$ where $x_{i} \geq 0$ for $i \geq l, \sum_{i=l}^{m} x_{i}=$ 1 and $\theta \in \mathbf{R}$.

Applying the method of Lagrange multipliers to the problem of maximizing $\left|P_{\alpha}\right|$ on the set $\mathbf{T}:=\left\{\left(0, \ldots, 0, x_{l}, \ldots, x_{m}\right): x_{i}>0\right.$ and $\left.\sum_{i=l}^{m} x_{i}=1\right\}$ yields the equations

$$
\sum_{i>l} x_{i}=\lambda=1-x_{l}
$$

and

$$
\left(\sum_{i=l}^{m} x_{i}\right)-x_{k}+\frac{\alpha_{k}-\alpha_{l}}{2 \alpha_{k}} 2 x_{k}=\lambda
$$

for $k>l$. Hence $\alpha_{l} x_{k}=\alpha_{k} x_{l}$ for $k \geq l$. Since $\sum_{i=l}^{m} x_{i}=\sum_{i=l}^{m} \alpha_{i}=1$ this implies $\alpha_{i}=x_{i}$ for $i \geq l$.

Hence

$$
\begin{aligned}
\sup \left\{\left|P_{\alpha}(x)\right|: x \in \mathbf{T}\right\} & =\sum_{l \leq i<j \leq m} \alpha_{i} \alpha_{j}+\sum_{k \geq l} \frac{\alpha_{k}-\alpha_{l}}{2 \alpha_{k}} \alpha_{k}^{2} \\
& =\frac{1}{2}\left(\sum_{i=l}^{m} \alpha_{i}\right)^{2}-\frac{1}{2} \alpha_{l} \cdot \sum_{k=l}^{m} \alpha_{k} \\
& =\frac{1}{2}\left(1-\alpha_{l}\right) .
\end{aligned}
$$


If $l^{*} \geq l,\left\{l^{*}\right\} \subset S \subset\left\{l^{*}, \ldots, m\right\}$ let

$$
\begin{aligned}
& \mathbf{S}=\left\{x \in \mathrm{R}^{m}: x=\left(0, \ldots, 0, x_{l^{*}}, \ldots, x_{m}\right),\right. \\
&\left.x_{i}>0 \text { if } i \in S, x_{i}=0 \text { if } i \notin S \text { and } \sum_{i=l}^{m} x_{i}=1\right\} .
\end{aligned}
$$

An analysis similar to the above shows that

$$
\sup \left\{\left|P_{\alpha}(x)\right|: x \in \mathbf{S}\right\}=\frac{\left(\sum_{i \in \mathbf{S}} \alpha_{i}\right)-\alpha_{l}}{2\left(\sum_{i \in \mathbf{S}} \alpha_{i}\right)}=\frac{1}{2}\left(1-\frac{\alpha_{l}}{\sum_{i \in \mathbf{S}} \alpha_{i}}\right)
$$

Since $\alpha_{i}>0$ when $i \geq l$ this shows that $\left\|P_{\alpha}\right\|=\left|P_{\alpha}\left(z_{1}, \ldots, z_{m}\right)\right|, \sum_{i=1}^{m}\left|z_{i}\right|=1$, if and only if $\left(z_{1}, \ldots, z_{m}\right)=e^{i \theta} \alpha$ for some $\theta \in \mathrm{R}$. Hence $\alpha^{2} \in \operatorname{Ext}_{R}\left(\mathscr{P}_{I}\left({ }^{2} \ell_{\infty}^{m}\right)\right)$ and we have established (3) when $n=2$.

If $\beta:=(1,1, \ldots, 1) \in \ell_{\infty}^{m}$ then $\|\beta\|=1$ and $\left\langle\beta,\left(z_{1}, \ldots, z_{m}\right)\right\rangle=\sum_{i=1}^{m} z_{i}$ for $\left(z_{1}, \ldots, z_{m}\right) \in \ell_{1}^{m}$. Hence $|\beta|$ achieves its maximum over the unit ball of $\ell_{1}^{m}$ precisely at all points of the form $e^{i \theta}\left(x_{1}, \ldots, x_{m}\right)$ where $x_{i} \geq 0, \sum_{i=1}^{m} x_{i}=1$ and $\theta \in \mathbf{R}$.

If $n>2$ then $Q:=P_{\alpha} \cdot \beta^{n-2}$ is an $n$-homogeneous polynomial on $\ell_{1}^{m}$ and $|Q|$ achieves its maximum over the unit ball of $\ell_{1}^{m}$ precisely at those points which have the form $e^{i \theta} \alpha$ where $\theta \in \mathrm{R}$ is arbitrary. Hence $\alpha^{n} \in$ $\operatorname{Ext}_{R}\left(\mathscr{P}_{I}\left({ }^{n} \ell_{\infty}^{m}\right)\right)$ and we have established (3) for all $n \geq 2$ and all $m$.

Note that the function

$$
f\left(\left(y_{n}\right)_{n}\right):=1+P_{\alpha}\left(\left(e^{i \theta_{n}} y_{\sigma(n)}\right)_{n=1}^{m}\right)
$$

satisfies $|f(y)|<|f(x)|$ for all $y \neq x$ in the closed unit ball of $\ell_{1}$. This may be used to show that all finitely supported unit vectors are peak points of different algebras of holomorphic functions (we refer to [2] for details).

\section{REFERENCES}

1. Aron, R. M., Choi, Y. S., Kim, S. G., Maestre, M., Local Properties of polynomials on a Banach space, Illinois J. Math. (to appear).

2. Aron, R. M., Choi, Y. S., Lourenço, M. 1., Paques, O. W., Boundaries for algebras of analytic functions on infinite dimensional Banach spaces, Contemp. Math. 144 (1993), 15-22.

3. Boyd, C., Ryan, R. A., Geometric theory of spaces of integral polynomials and symmetric tensor products, J. Funct. Anal. 179 (2001), 18-42.

4. Braun, R., Kaup, W., Upmeier, H., A holomorphic characterization of Jordan $\mathscr{C}^{*}$-algebras, Math. Z. 161 (1978), 277-290.

5. Braun, R., Kaup, W., Upmeier, H., On the automorphisms of circular and Reinhardt domains in complex Banach spaces, Manuscripta Math. 25 (1978), 97-133. 
6. Carando, D., Dimant, V., Duality in spaces of nuclear and integral polynomials, J. Math. Anal. Appl. 241 (2000), 107-121.

7. Choi, Y. S., Kim, S. G., The unit ball of $\mathscr{P}\left({ }^{2} \ell_{2}^{2}\right)$, Arch. Math. 71 (1998), 472-480.

8. Choi, Y. S., Kim, S. G., Smooth points of the unit ball of the space $\mathscr{P}\left({ }^{2} \ell_{1}\right)$, Resultate Math. 36 (1999), 26-33.

9. Diestel, J., Sequences and series in Banach spaces, Graduate Texts in Math. 92 (1984).

10. Deville, R., Godefroy, G., Zizler, V., Smoothness and renorming in Banach spaces, Pitman Monographs Surveys Pure Appl. Math. 64 (1993).

11. Dineen, S., Complex Analysis on Infinite Dimensional Spaces, Monographs Math., 1999.

12. Grecu, B., Geometry of 3-homogeneous polynomials on real Hilbert spaces, J. Math. Anal. Appl. 246 (2000), 217-229.

13. Grecu, B., Smooth 2-homogeneous polynomials on Hilbert spaces, Arch Math. 76 (2001), 445-454.

14. Harris, L. A., Schwarz's lemma and the maximum principle in infinite dimensional spaces, Thesis, Cornell University, Ithaca, N.Y., 1969.

15. Harris, L. A., Bounded symmetric domains in infinite dimensional spaces, Infinite Dimensional Holomorphy, Eds. T. L. Hayden, T. J. Suffridge, Lecture Notes in Math. 365 (1974), $1-15$.

16. Kadison, R. V., Isometries of operator algebras, Ann. of Math. 54 (1959), 325-338.

17. Murphy, G. J., $\mathscr{C}^{*}$-Algebras and Operator theory, Academic Press, 1990.

18. Ryan, R. A., Turett, B., Geometry of spaces of polynomials, J. Math. Anal. Appl. 221 (1998), 698-711.

19. Smul'yan, V. L., On some geometrical properties of the unit sphere of spaces of type (B), Mat. Sb. (N.S.) 6, no. 48 (1939), 77-94.

20. Sundaresan, K., Geometry of spaces of quadratic forms, Inner Product Spaces and Applications, Pitman Res. Notes Math. Ser. 376 (1997), 47-49.

21. Thorpe, E., Whitley, R., The strong maximum modulus theorem for analytic functions into a Banach space, Proc. Amer. Math. Soc. 18 (1967), 640-646.

22. Vesentini, E., Complex geodesics and holomorphic mappings, Sympos. Math. 26 (1982), 211-230.

23. Vigué, J. P., Sur la caractérisation des isomorphismes analytiques entre domaines bornés d'un espace de Banach complexe, Ann. Scuola Norm. Sup. Pisa Cl. Sci. (4) 21 (1994), 145-155.

DEPARTMENT OF MATHEMATICS

UNIVERSITY COLLEGE DUBLIN

BELFIELD, DUBLIN 4

IRELAND 